\title{
Association of maternal dietary patterns with child birth weight and size in India: Evidence from National Family Health Survey, 2005 to 2016
}

\author{
Brajesh $^{1}$, Chander Shekhar ${ }^{2}$ \\ From ${ }^{1}$ PhD Scholar, International Institute for Population Sciences, Mumbai, Maharashtra, India, ${ }^{2}$ Professor, Department of Fertility and Social \\ Demography, International Institute for Population Sciences, Mumbai, Maharashtra, India
}

\begin{abstract}
Background: Maternal dietary diversity during pregnancy is a major determinant of birth weight and birth size of infant. During pregnancy, mother diet is highly dependent on their topographical, cultural, and sociodemographic circumstances. Objective: The objective of the study was to assess the association between the maternal dietary intake with child birth weight and birth size along with the sociodemographic factors in India. Materials and Methods: The data for this study were drawn from the National Family Health Survey (NFHS), 2005 to 2006 and 2015 to 2016 held in India. Inferential statistical analysis Chi-square was built to test was used to examine the association between maternal dietary patterns, and logistic regression model was used to analyze the effect of mother's dietary patterns on child birth weight and size by controlling the sociodemographic factors. Results: Mother's daily intake of fish, meat, green leafy vegetables, and fruits was associated with higher birth weight or size and lower risk of intrauterine growth retardation. Women with $\geq 2$ dietary diversity categories had significantly higher proportion of low birth weight (LBW) and size of babies comparatively to those in the $\geq 2$ dietary diversity categories, there were lesser chance of LBW and small size of babies with women's dietary diversity 3 (odds ranges from 1.09 to 1.44 ) or $\geq 4$ (odds ranges from 1.15 to 1.59 ). Furthermore, low birth order, mother's underweight status, low maternal education, and wealth status significantly have positive association with the poor birth outcomes. Conclusion: The birth weight and size of newborns were found positively associated with the mother's dietary intake. To meet the aim of maternal dietary diversity and to achieve the double bonus, the government should focus more on supplementation and food security programs during pregnancy that also include nutritional education as well as behavioral and social change interventions strategies.
\end{abstract}

Key words: Birth size, Birth weight, India, Maternal dietary diversity, Newborn

$\mathrm{D}$ uring pregnancy, it is essential for women to have nutritional diet with supplements to maintain maternal health and to allow the adequate fetal growth and development. India has witnessed a high burden of childhood malnutrition and mortality [1,2]. Although, India has made considerable progress in reducing low birth weight (LBW) of children during over the past decades, still remains a leading cause of child mortality in the country, especially among the socioeconomically disadvantageous groups [3]. According to the latest National Family Health Survey (NFHS)-4, about 18\% of Indian children younger than 5 years were born with LBW in 2015-2016 [4]. Birth weight and birth size are important indicators of child survival and reflect the risk to child death and health hazards; especially, for the first few years of life. Several factors are related to variations in birth weight and birth

\section{Access this article online}

Received - 05 December 2021

Initial Review - 10 December 2021

Accepted - 12 December 2021

DOI: $10.32677 /$ ijch.v8i12.3237 size including maternal food consumption. However, assessing the food consumption becomes a challenge due to the human diet complexity, something that solicits researchers to refine assessment methods of mother's food consumption [5-7]. Despite increased interest in promoting nutrition during pregnancy, the association between maternal dietary patterns and birth outcomes has been equivocal $[8,9]$. Some of the researchers found that maternal motivation could change to eat have also been associated with increased child food intake and infant birth weight and size. However, very few studies have detected an association between maternal diet pattern during pregnancy with birth weight and size of the baby $[7,10,11]$. Maternal nutrition has a direct effect on the birth weight and size of the new born, as less nourished mothers are found to deliver higher percentage of LBW or small size of babies as compared to mother who are well nourished [12]. Both mother and child are believed as a single unit whether it is socially, culturally, or most importantly biologically. A child received a

Correspondence to: Brajesh, PhD Scholar, International Institute for Population Sciences, Deonar, Govandi Station Road, Mumbai - 400088, Maharashtra, India. E-mail: iipsmumbai2012@gmail.com

(C) 2021 Creative Commons Attribution-NonCommercial 4.0 International License (CC BY-NC-ND 4.0). 
biological support from its mother in the course of its development and growth throughout the period of pregnancy and lactation, and it totally depends on quality and quantity of nourishment a mother received during her pregnancy period $[8,13]$. Pregnancy outcome is directly linked to the status of maternal nutrition because lower calorie and protein intake by a mother throughout the pregnancy can result in LBW or small size of baby. The study assumes that mothers were taking same foods items during their pregnancy and lactation period, as available in the Demographic Health Survey. Hence, this study aimed to examine the relationship between the mother's diet pattern with birth weight and size of the newborn in India between 2005-2006 and 2015-2016.

\section{MATERIALS AND METHODS}

Full retrospective maternity birth history data of India from two survey recent rounds of NFHS, NFHS-3 (2005-2006), and NFHS-4 (2015-2016) have been utilized for the study. NFHS-3 captures the information of 256,782 births which occurred to 124,385 women aged 15-49 years between 1968 and 2006 . NFHS-4 has information about 1,315,617 births which were nested within 699,686 mothers in the age of 15-49 years between 1970 and 2016. In both the two survey rounds, different information related to all births, mother's diet practices, birth weight, birth size, and other sociodemographic predictors are available for a period of spanning nearly 50 years which provide an opportunity to examine association between diet pattern of mother and birth outcome. The present study assumed that the mothers consumed the same food items available at the time of the survey during pregnancy and lactation. We calculated the dietary diversity based on the number of food groups consumed during pregnancy by women on daily, weekly, or occasionally basis. The outcome variables have been categorized as in two groups; the birth weight of the infant in grams coded as a dichotomous variable, which takes the value " 1 " if the infant weight more than $2500 \mathrm{~g}$ and " 0 " if infant birth weight is $<2500 \mathrm{~g}$. Infant's size at birth was also used as a binary outcome variable: "Small size" and " $\geq$ average size." Small size (coded $=0$ ) consists of "very small and smaller than average size," while $\geq$ average size (coded=1) consists of "very large, larger than average and average size." Predictors variables were taken foods items: The categorical responses of various food intake frequencies such as occasionally, daily, and weekly were given in the data whereas never and occasionally have recoded into one category due to fewer cases, other covariates such as education of mother, women's empowerment level, household wealth status, caste, residence, religion, family size, and institutional delivery. We used principal component analysis for the responses of decision-making questions related to women's empowerment, where the resulted scores were categorized into three groups: Low, medium, and high. Association between sociodemographic status and mother's diet pattern with birth weight and size of infants was showed using the Pearson's Chisquared statistic. Furthermore, binary logistic regression (BLR) models were employed to assess the effect of maternal diet pattern on the birth weight and size. The collinearity test was conducted using variance inflation factor technique between all the selected explanatory variables. We have found no collinearity in these variables. The regression results are presented by estimated odds ratios with $95 \%$ confidence intervals. All the statistical analyses were performed using the STATA version 14.

\section{RESULTS}

Association between Mother's Diet Pattern with Birth Weight and Birth Size

In both the NFHS surveys, over four-fifths of women reported daily consumption of foods to normal birth weight of their infants. NFHS-3 had a higher proportion of female children with LBW than NFHS-4. Approximately four-fifths of children in the second and higher birth orders categories had LBW. The child's birth weight was also associated to the mother's age. During the NFHS-3, three-quarters of mothers with a primary education and four-fifths of mothers with a secondary education had normal child birth weight. Similar results are continued in the NFHS-4 that there was no difference in the categories. In the high and medium empowered groups of women, the proportion of children with normal birth weight was higher. Other castes and other backward classes (OBCs) accounted for over three-quarters of children with normal birth weight in NFHS-3 and nearly four-fifths in NFHS-4. Smaller families had a higher proportion of children with normal birth weight than larger families. In both NFHS-3 and NFHS4 , most of the normal birth weight babies were born in healthcare facilities; private hospitals (four-fifths) had the highest contribution. Most of the background characteristics of mothers were found to strongly relate to birth weight and size indices.

In both the surveys, four-fifths of babies were born with birth weights higher than the average birth weight. Children had normal birth size (average or more), in 2005-2006 (81\%) and 2015$2016(76 \%)$ among three-quarters of mothers who had consumed fruits and eggs daily. In 2005-2006, about three-fifths of twins had an above average birth size, compared to four-fifths in 2015-2016. In NFHS-3, more than three-quarters of normal BMI mothers and $82 \%$ of obese mothers had newborns with the average or above birth size, while in NFHS- $4,89 \%$ and $77 \%$ had newborns with the average or above birth size. This was a significant finding that four-fifths of children born to mothers aged 25 years and above were of average or large size at birth. A similar proportion of children born to mothers with secondary or higher education followed by primary education was of average or larger birth size. Between NFHS-3 and NFHS-4, the proportion of children of average or above average birth size born to women of high empowerment decreased slightly. The average and above birth size was found in less than four-fifths of babies born in families with nine or more members followed by 5-8 members, and it has improved during the two survey periods. In 2015-2016, a higher proportion of children born in any type of health facility were measured at average or larger birth size than in 2005-2006. Across the most socioeconomic and demographic factors, mothers' food intake has increased over the last decade (Table 1). 
Table 1: Percentage distribution of birth weight and birth size by mother's food intake and sociodemographic factors in India, $2005-2016$

\begin{tabular}{|c|c|c|c|c|c|c|c|c|c|}
\hline \multirow{3}{*}{$\begin{array}{l}\text { Background } \\
\text { characteristics }\end{array}$} & \multirow[t]{3}{*}{ Category } & \multicolumn{4}{|c|}{ Birth weight } & \multicolumn{4}{|c|}{ Birth size } \\
\hline & & \multicolumn{2}{|c|}{$\begin{array}{c}\text { NFHS-3 } \\
(2005-06)\end{array}$} & \multicolumn{2}{|c|}{$\begin{array}{c}\text { NFHS-4 } \\
(2015-16)\end{array}$} & \multicolumn{2}{|c|}{$\begin{array}{c}\text { NFHS-3 } \\
(2005-06)\end{array}$} & \multicolumn{2}{|c|}{$\begin{array}{c}\text { NFHS-4 } \\
(2015-16)\end{array}$} \\
\hline & & $<2499 \mathrm{~g}$ & $\geq 2500 \mathrm{~g}$ & $<2499 \mathrm{~g}$ & $\geq 2500 \mathrm{~g}$ & $<$ Average & $\geq$ Average & $<$ Average & $\geq$ Average \\
\hline \multirow[t]{4}{*}{ Milk and curd } & Never/occasionally & 24.0 & 76.0 & 19.5 & 80.5 & 23.7 & 76.4 & 16.6 & 83.4 \\
\hline & Daily & 19.6 & 80.4 & 17.1 & 82.9 & 20.5 & 79.6 & 21.5 & 78.5 \\
\hline & Weekly & 22.0 & 78.0 & 18.7 & 81.3 & 21.3 & 78.7 & 20.1 & 79.9 \\
\hline & & \multicolumn{2}{|c|}{$\begin{array}{c}\chi^{2}=20.867 \\
p \leq 0.001\end{array}$} & \multicolumn{2}{|c|}{$\begin{array}{c}\chi^{2}=34.833 \\
\mathrm{p} \leq 0.001\end{array}$} & \multicolumn{2}{|c|}{$\begin{array}{c}\chi^{2}=91.738 \\
p \leq 0.001\end{array}$} & \multicolumn{2}{|c|}{$\begin{array}{c}\chi^{2}=411.993 \\
\mathrm{p} \leq 0.001\end{array}$} \\
\hline \multirow[t]{4}{*}{ Pulses and beans } & Never/occasionally & 22.2 & 77.8 & 20.7 & 79.4 & 28.7 & 71.3 & 17.0 & 83.1 \\
\hline & Daily & 20.4 & 79.6 & 17.0 & 83.0 & 20.3 & 79.7 & 20.2 & 79.8 \\
\hline & Weekly & 23.3 & 76.7 & 18.9 & 81.1 & 22.9 & 77.1 & 19.3 & 80.7 \\
\hline & & \multicolumn{2}{|c|}{$\begin{array}{c}\chi^{2}=9.328 \\
\mathrm{p} \leq 0.009\end{array}$} & \multicolumn{2}{|c|}{$\begin{array}{c}\chi^{2}=86.361 \\
p \leq 0.001\end{array}$} & \multicolumn{2}{|c|}{$\begin{array}{c}\chi^{2}=171.305 \\
p \leq 0.001\end{array}$} & \multicolumn{2}{|c|}{$\begin{array}{c}\chi^{2}=286.466 \\
p \leq 0.001\end{array}$} \\
\hline \multirow{4}{*}{$\begin{array}{l}\text { Green leafy } \\
\text { vegetables }\end{array}$} & Never/occasionally & 23.0 & 77.0 & 21.0 & 79.0 & 24.4 & 75.6 & 14.5 & 85.6 \\
\hline & Daily & 21.2 & 78.8 & 17.2 & 82.8 & 21.8 & 78.2 & 20.8 & 79.2 \\
\hline & Weekly & 22.2 & 77.8 & 18.5 & 81.5 & 22.3 & 77.7 & 19.9 & 80.2 \\
\hline & & \multicolumn{2}{|c|}{$\begin{array}{c}\chi^{2}=17.645 \\
p \leq 0.001\end{array}$} & \multicolumn{2}{|c|}{$\begin{array}{c}\chi^{2}=30.371 \\
\mathrm{p} \leq 0.001\end{array}$} & \multicolumn{2}{|c|}{$\begin{array}{l}\chi^{2}=8.152 \\
\mathrm{p} \leq 0.017\end{array}$} & \multicolumn{2}{|c|}{$\begin{array}{c}\chi^{2}=925.441 \\
\mathrm{p} \leq 0.001\end{array}$} \\
\hline \multirow[t]{4}{*}{ Fruits } & Never/occasionally & 24.6 & 75.4 & 19.1 & 80.9 & 23.2 & 76.8 & 17.2 & 82.8 \\
\hline & Daily & 18.4 & 81.6 & 16.2 & 83.8 & 18.5 & 81.5 & 23.7 & 76.4 \\
\hline & Weekly & 19.2 & 80.8 & 17.5 & 82.5 & 20.5 & 79.5 & 22.4 & 77.6 \\
\hline & & $\begin{array}{r}\chi^{2}=7 \\
\mathrm{p} \leq\end{array}$ & $\begin{array}{l}.041 \\
001\end{array}$ & $\begin{array}{r}\chi^{2}=11 \\
\mathrm{p} \leq 0\end{array}$ & $\begin{array}{l}113 \\
001\end{array}$ & $\begin{array}{r}\chi^{2}=13 \\
p \leq 0\end{array}$ & .254 & $\begin{array}{r}\chi^{2}=90 \\
p \leq 0\end{array}$ & $\begin{array}{l}.717 \\
001\end{array}$ \\
\hline Eggs & Never/occasionally & 22.6 & 77.5 & 19.0 & 81.1 & 22.8 & 77.2 & 17.1 & 82.9 \\
\hline & Daily & 18.5 & 81.5 & 16.6 & 83.4 & 21.5 & 78.5 & 25.5 & 74.5 \\
\hline & Weekly & 20.4 & 79.6 & 17.3 & 82.7 & 20.5 & 79.5 & 22.9 & 77.1 \\
\hline & & $\begin{array}{r}\chi^{2}=3 \\
\mathrm{p} \leq\end{array}$ & $\begin{array}{l}347 \\
001\end{array}$ & $\begin{array}{r}\chi^{2}=14 \\
p \leq 0\end{array}$ & $\begin{array}{l}.081 \\
001\end{array}$ & $\begin{array}{r}\chi^{2}=3 \\
p \leq 1\end{array}$ & $\begin{array}{l}.716 \\
001\end{array}$ & $\begin{array}{r}\chi^{2}=10 \\
p \leq 0\end{array}$ & $\begin{array}{l}0.12 \\
001\end{array}$ \\
\hline Fish & Never/occasionally & 21.8 & 78.2 & 18.7 & 81.3 & 22.1 & 77.9 & 17.6 & 82.4 \\
\hline & Daily & 17.6 & 82.4 & 16.3 & 83.7 & 20.2 & 79.8 & 25.4 & 74.6 \\
\hline & Weekly & 22.2 & 77.8 & 17.4 & 82.6 & 22.8 & 77.2 & 23.1 & 76.9 \\
\hline & & $\begin{array}{c}\chi^{2}=1 \\
\mathrm{p} \leq\end{array}$ & $\begin{array}{l}123 \\
001\end{array}$ & $\begin{array}{r}\chi^{2}=8 \\
p \leq 0\end{array}$ & $\begin{array}{l}678 \\
001\end{array}$ & $\begin{array}{r}\chi^{2}=8 \\
p \leq 1\end{array}$ & $\begin{array}{l}454 \\
016\end{array}$ & $\begin{array}{l}\chi^{2}= \\
\mathrm{p} \leq \mathrm{c}\end{array}$ & $\begin{array}{l}400 \\
001\end{array}$ \\
\hline Meat & Never/occasionally & 22.0 & 78.0 & 18.7 & 81.3 & 22.6 & 77.4 & 17.8 & 82.2 \\
\hline & Daily & 20.6 & 79.4 & 16.9 & 83.1 & 23.5 & 76.5 & 25.1 & 74.9 \\
\hline & Weekly & 20.5 & 79.5 & 17.2 & 82.8 & 20.3 & 79.7 & 23.1 & 77.0 \\
\hline & & $\begin{array}{c}\chi^{2}=3 \\
\mathrm{p} \leq\end{array}$ & $\begin{array}{l}532 \\
001\end{array}$ & $\begin{array}{r}\chi^{2}=12 \\
\mathrm{p} \leq 0\end{array}$ & $\begin{array}{l}953, \\
001\end{array}$ & $\begin{array}{r}\chi^{2}=3 \\
\mathrm{p} \leq 1\end{array}$ & $\begin{array}{l}935 \\
001\end{array}$ & $\begin{array}{r}\chi^{2}=82 \\
\mathrm{p} \leq 0\end{array}$ & $\begin{array}{l}.299 \\
001\end{array}$ \\
\hline Sex of child & Male & 20.3 & 79.7 & 17.1 & 82.9 & 21.2 & 78.8 & 20.0 & 80.0 \\
\hline & Female & 23.0 & 77.0 & 19.4 & 80.6 & 23.2 & 76.8 & 18.9 & 81.1 \\
\hline & & $\begin{array}{r}\chi^{2}=1 \\
\mathrm{p} \leq\end{array}$ & $\begin{array}{l}0334, \\
001\end{array}$ & $\begin{array}{r}\chi^{2}=16 \\
p \leq 0\end{array}$ & $\begin{array}{l}.504, \\
001\end{array}$ & $\begin{array}{r}\chi^{2}=32 \\
p \leq 0\end{array}$ & $\begin{array}{l}0627, \\
001\end{array}$ & $\begin{array}{c}\chi^{2}=3 \\
\mathrm{p} \leq 0\end{array}$ & $\begin{array}{l}618 \\
001\end{array}$ \\
\hline Twin child & No & 20.5 & 79.5 & 64.1 & 35.9 & 21.8 & 78.17 & 19.5 & 80.5 \\
\hline & Yes & 74.6 & 25.4 & 17.4 & 82.6 & 42.3 & 57.7 & 16.2 & 83.8 \\
\hline & & $\begin{array}{r}\chi^{2}=6 \\
p \leq\end{array}$ & $\begin{array}{l}.016, \\
001\end{array}$ & $\begin{array}{r}\chi^{2}=25 \\
\mathrm{p} \leq 0\end{array}$ & $\begin{array}{l}104 \\
001\end{array}$ & $\begin{array}{r}\chi^{2}=2 \\
\mathrm{p} \leq 1\end{array}$ & $\begin{array}{l}.195 \\
001\end{array}$ & $\begin{array}{r}\chi^{2}=25 \\
p \leq 0\end{array}$ & $\begin{array}{l}.104, \\
001\end{array}$ \\
\hline Birth order & $1^{\text {st }}$ & 22.4 & 77.7 & 19.0 & 81.0 & 22.9 & 77.1 & 20.4 & 79.7 \\
\hline & $2^{\text {nd }}-3^{\text {rd }}$ & 20.2 & 79.8 & 17.3 & 82.7 & 21.4 & 78.6 & 19.9 & 80.1 \\
\hline & $4^{\text {th }}$ and above & 22.4 & 77.7 & 19.0 & 81.0 & 22.9 & 77.1 & 20.4 & 79.7 \\
\hline & & $\begin{array}{c}\chi^{2}=7 \\
p \leq\end{array}$ & $\begin{array}{l}896, \\
024\end{array}$ & $\begin{array}{r}\chi^{2}=10 \\
p \leq 0\end{array}$ & $\begin{array}{l}.165 \\
001\end{array}$ & $\begin{array}{c}\chi^{2}=2 \\
p \leq 1\end{array}$ & $\begin{array}{l}.957, \\
001\end{array}$ & $\begin{array}{r}\chi^{2}=17 \\
p \leq c\end{array}$ & $\begin{array}{l}.275 \\
001\end{array}$ \\
\hline
\end{tabular}


Table 1: (Continued)

\begin{tabular}{|c|c|c|c|c|c|c|c|c|c|c|}
\hline \multirow{3}{*}{$\begin{array}{l}\text { Background } \\
\text { characteristics }\end{array}$} & \multirow[t]{3}{*}{ Category } & \multicolumn{4}{|c|}{ Birth weight } & \multicolumn{5}{|c|}{ Birth size } \\
\hline & & \multicolumn{2}{|c|}{$\begin{array}{c}\text { NFHS-3 } \\
(2005-06)\end{array}$} & \multicolumn{2}{|c|}{$\begin{array}{c}\text { NFHS-4 } \\
(2015-16)\end{array}$} & \multicolumn{3}{|c|}{$\begin{array}{c}\text { NFHS-3 } \\
(2005-06)\end{array}$} & \multicolumn{2}{|c|}{$\begin{array}{c}\text { NFHS-4 } \\
(2015-16)\end{array}$} \\
\hline & & $<2499 \mathrm{~g}$ & $\geq 2500 \mathrm{~g}$ & $<2499 \mathrm{~g}$ & $\geq 2500 \mathrm{~g}$ & $<$ Average & $\geq$ Ave & rage & $<$ Average & $\geq$ Average \\
\hline \multirow[t]{4}{*}{ BMI of women } & Underweight & 25.6 & 74.4 & 21.7 & 78.4 & 24.4 & & 75.6 & 17.8 & 82.2 \\
\hline & Normal & 20.7 & 79.3 & 17.6 & 82.4 & 21.4 & & 78.6 & 19.2 & 80.8 \\
\hline & Overweight/obese & 17.2 & 82.8 & 15.6 & 84.4 & 17.8 & & 82.2 & 23.0 & 77.0 \\
\hline & & \multicolumn{2}{|c|}{$\begin{array}{c}\chi^{2}=113.061 \\
\mathrm{p} \leq 0.000\end{array}$} & \multicolumn{2}{|c|}{$\begin{array}{c}\chi^{2}=629.384 \\
\mathrm{p} \leq 0.001\end{array}$} & \multicolumn{3}{|c|}{$\begin{array}{c}\chi^{2}=153.542 \\
p \leq 0.000\end{array}$} & \multicolumn{2}{|c|}{$\begin{array}{c}\chi^{2}=263.557 \\
p \leq 0.000\end{array}$} \\
\hline \multirow[t]{4}{*}{ Age of mother } & 15-24 years & 24.0 & 76.0 & 19.7 & 80.3 & 24.0 & & 76.0 & 20.2 & 79.8 \\
\hline & 25-34 years & 19.9 & 80.1 & 17.3 & 82.7 & 20.7 & & 79.3 & 19.3 & 80.7 \\
\hline & 35 and above years & 16.8 & 83.3 & 17.9 & 82.1 & 21.3 & & 78.7 & 17.8 & 82.2 \\
\hline & & \multicolumn{2}{|c|}{$\begin{array}{c}\chi^{2}=75.315 \\
\mathrm{p} \leq 0.001\end{array}$} & \multicolumn{2}{|c|}{$\begin{array}{c}\chi^{2}=248.044 \\
p \leq 0.001\end{array}$} & \multicolumn{3}{|c|}{$\begin{array}{c}\chi^{2}=125.354 \\
p \leq 0.001\end{array}$} & \multicolumn{2}{|c|}{$\begin{array}{c}\chi^{2}=16.191, \\
p \leq 0.001\end{array}$} \\
\hline \multirow[t]{4}{*}{ Education } & Illiterate & 26.2 & 73.8 & 20.1 & 79.9 & 23.7 & & 76.3 & 16.9 & 83.1 \\
\hline & Primary & 24.6 & 75.5 & 20.2 & 79.9 & 23.4 & & 76.6 & 19.0 & 81.0 \\
\hline & Secondary and above & 19.5 & 80.5 & 17.2 & 82.9 & 19.5 & & 80.5 & 21.0 & 79.0 \\
\hline & & \multicolumn{2}{|c|}{$\begin{array}{c}\chi^{2}=128.499 \\
p \leq 0.001\end{array}$} & \multicolumn{2}{|c|}{$\begin{array}{c}\chi^{2}=347.042 \\
p \leq 0.001\end{array}$} & \multicolumn{3}{|c|}{$\begin{array}{c}\chi^{2}=252.447 \\
\mathrm{p} \leq 0.001\end{array}$} & \multicolumn{2}{|c|}{$\begin{array}{c}\chi^{2}=325.867 \\
p \leq 0.001\end{array}$} \\
\hline \multirow{4}{*}{$\begin{array}{l}\text { Empowerment } \\
\text { level }\end{array}$} & Low & 23.2 & 76.8 & 19.7 & 80.3 & 24.7 & & 75.3 & 21.2 & 78.8 \\
\hline & Medium & 22.6 & 77.4 & 17.3 & 82.7 & 22.0 & & 78.0 & 21.0 & 79.0 \\
\hline & High & 19.1 & 80.9 & 17.9 & 82.1 & 19.6 & & 80.5 & 17.2 & 82.8 \\
\hline & & $\begin{array}{r}\chi^{2}=1 \\
\mathrm{p} \leq 0\end{array}$ & $\begin{array}{l}225, \\
01\end{array}$ & $\begin{array}{r}\chi^{2}=2 \\
p \leq 0\end{array}$ & $\begin{array}{l}3.044, \\
001\end{array}$ & $\begin{array}{r}\chi^{2}=10 \\
p \leq 1\end{array}$ & $\begin{array}{l}7.109, \\
001\end{array}$ & & $\begin{array}{r}\chi^{2}=38 \\
p \leq 0\end{array}$ & $\begin{array}{l}0345, \\
001\end{array}$ \\
\hline Wealth index & Poorest & 25.4 & 74.6 & 19.7 & 80.3 & 25.3 & & 74.7 & 16.9 & 83.1 \\
\hline & Poorer & 25.4 & 74.6 & 19.1 & 80.9 & 23.7 & & 76.3 & 19.0 & 81.0 \\
\hline & Middle & 23.7 & 76.3 & 18.7 & 81.3 & 22.4 & & 77.6 & 21.0 & 79.0 \\
\hline & Richer & 21.8 & 78.2 & 18.1 & 81.9 & 19.8 & & 80.2 & 21.2 & 78.9 \\
\hline & Richest & 17.4 & 82.6 & 15.1 & 84.9 & 16.9 & & 83.1 & 20.5 & 79.5 \\
\hline & & $\begin{array}{r}\chi^{2}=10 \\
\mathrm{p} \leq 0\end{array}$ & 901 & $\begin{array}{r}\chi^{2}=26 \\
\mathrm{p} \leq 0\end{array}$ & $\begin{array}{l}9.978 \\
001\end{array}$ & $\begin{array}{r}\chi^{2}=42 \\
\mathrm{p} \leq 1\end{array}$ & $\begin{array}{l}7.621 \\
001\end{array}$ & & $\begin{array}{r}\chi^{2}=25 \\
\mathrm{p} \leq 0\end{array}$ & $\begin{array}{l}.604, \\
001\end{array}$ \\
\hline Caste & $\mathrm{SC} / \mathrm{ST}$ & 23.3 & 76.7 & 19.6 & 80.4 & 23.6 & & 76.4 & 19.1 & 80.9 \\
\hline & $\mathrm{OBC}$ & 21.3 & 78.7 & 17.7 & 82.3 & 21.4 & & 78.6 & 19.2 & 80.8 \\
\hline & Other & 20.8 & 79.2 & 17.4 & 82.7 & 21.4 & & 78.6 & 19.9 & 80.1 \\
\hline & & $\begin{aligned} \chi^{2} & =0 \\
p & \leq 0\end{aligned}$ & $\begin{array}{l}340, \\
627\end{array}$ & & $\begin{array}{l}005 \\
030\end{array}$ & $\begin{array}{c}\chi^{2}=4 \\
\mathrm{p} \leq\end{array}$ & $\begin{array}{l}.098 \\
000\end{array}$ & & $\begin{array}{c}\chi^{2}=8 \\
\mathrm{p} \leq 0\end{array}$ & $\begin{array}{l}465 \\
017\end{array}$ \\
\hline Residence & Rural & 23.3 & 76.7 & 18.5 & 81.5 & 22.9 & & 77.1 & 18.9 & 81.1 \\
\hline & Urban & 19.3 & 80.7 & 17.6 & 82.4 & 19.8 & & 80.2 & 21.0 & 79.1 \\
\hline & & $\begin{array}{r}\chi^{2}=2 \\
\mathrm{p} \leq 0\end{array}$ & $\begin{array}{l}728, \\
01\end{array}$ & $\begin{array}{c}\chi^{2}=1 \\
\mathrm{p} \leq 1\end{array}$ & $\begin{array}{l}.038 \\
001\end{array}$ & $\begin{array}{r}\chi^{2}=1 \\
\mathrm{p} \leq 1\end{array}$ & $\begin{array}{l}59.3 \\
001\end{array}$ & & $\begin{aligned} \chi^{2} & =0 \\
\mathrm{p} & \leq 0\end{aligned}$ & $\begin{array}{l}.86, \\
001\end{array}$ \\
\hline Religion & Hindu & 21.8 & 78.2 & 18.5 & 81.5 & 22.0 & & 78.0 & 19.5 & 80.5 \\
\hline & Muslims & 20.2 & 79.8 & 17.3 & 82.7 & 22.5 & & 77.5 & 19.5 & 80.5 \\
\hline & Other & 20.8 & 79.2 & 16.7 & 83.3 & 22.9 & & 77.1 & 19.8 & 80.2 \\
\hline & & $\begin{array}{r}\chi^{2}=6 \\
\mathrm{p} \leq 0\end{array}$ & $\begin{array}{l}6.42, \\
01\end{array}$ & $\begin{array}{c}\chi^{2}=6 \\
p \leq c\end{array}$ & $\begin{array}{l}6.42 \\
001\end{array}$ & $\begin{array}{l}\chi^{2}=3 \\
\mathrm{p} \leq 0\end{array}$ & $\begin{array}{l}939, \\
140\end{array}$ & & $\begin{array}{r}\chi^{2}=2 \\
\mathrm{p} \leq 0\end{array}$ & $\begin{array}{l}451 \\
001\end{array}$ \\
\hline Family size & $0-4$ members & 21.0 & 79.0 & 18.2 & 81.8 & 23.0 & & 77.0 & 20.9 & 79.1 \\
\hline & 5-8 members & 21.7 & 78.3 & 18.2 & 81.8 & 22.1 & & 77.9 & 19.2 & 80.8 \\
\hline & $9+$ members & 22.0 & 78.0 & 18.2 & 81.8 & 21.4 & & 78.6 & 18.6 & 81.4 \\
\hline & & $\begin{aligned} \chi^{2} & =7 . \\
p & \leq 0\end{aligned}$ & & $\begin{array}{c}\chi^{2}=7 \\
p \leq c\end{array}$ & $\begin{array}{l}484, \\
024\end{array}$ & $\begin{array}{c}\chi^{2}=1 \\
p \leq 1\end{array}$ & $\begin{array}{l}.152 \\
002\end{array}$ & & $\begin{array}{c}\chi^{2}=5 \\
p \leq 0\end{array}$ & $\begin{array}{l}906, \\
001\end{array}$ \\
\hline
\end{tabular}

(Contd...) 
Table 1: (Continued)

\begin{tabular}{|c|c|c|c|c|c|c|c|c|c|}
\hline \multirow{3}{*}{$\begin{array}{l}\text { Background } \\
\text { characteristics }\end{array}$} & \multirow[t]{3}{*}{ Category } & \multicolumn{4}{|c|}{ Birth weight } & \multicolumn{4}{|c|}{ Birth size } \\
\hline & & \multicolumn{2}{|c|}{$\begin{array}{c}\text { NFHS-3 } \\
(2005-06)\end{array}$} & \multicolumn{2}{|c|}{$\begin{array}{c}\text { NFHS-4 } \\
(2015-16)\end{array}$} & \multicolumn{2}{|c|}{$\begin{array}{c}\text { NFHS-3 } \\
(2005-06)\end{array}$} & \multicolumn{2}{|c|}{$\begin{array}{c}\text { NFHS-4 } \\
(2015-16)\end{array}$} \\
\hline & & $<2499 \mathrm{~g}$ & $\geq 2500 \mathrm{~g}$ & $<2499 \mathrm{~g}$ & $\geq 2500 \mathrm{~g}$ & $<$ Average & $\geq$ Average & $<$ Average & $\geq$ Average \\
\hline \multirow{4}{*}{$\begin{array}{l}\text { Institutional } \\
\text { delivery }\end{array}$} & Public hospital & 22.1 & 77.9 & 18.2 & 81.8 & 21.8 & 78.2 & 19.8 & 80.2 \\
\hline & Private hospital & 20.3 & 79.7 & 17.7 & 82.3 & 19.8 & 80.2 & 21.8 & 78.2 \\
\hline & Home & 24.0 & 76.0 & 20.4 & 79.6 & 23.1 & 76.9 & 15.7 & 84.3 \\
\hline & & \multicolumn{2}{|c|}{$\begin{array}{c}\chi^{2}=8.979 \\
\mathrm{p} \leq 0.001\end{array}$} & \multicolumn{2}{|c|}{$\begin{array}{c}\chi^{2}=52.269, \\
p \leq 0.001\end{array}$} & \multicolumn{2}{|c|}{$\begin{array}{c}\chi^{2}=121.478 \\
p \leq 0.001\end{array}$} & \multicolumn{2}{|c|}{$\begin{array}{c}\chi^{2}=566.114 \\
p \leq 0.001\end{array}$} \\
\hline
\end{tabular}

NFHS-5: National Family Health Survey-5, BMI: Body mass index, SC: Scheduled caste, ST: Scheduled tribe, OBC: Other backward class, g: Grams

Effect of Mother's Food Intake on Birth Weight and Birth Size

\section{Birth weight}

Tables 2 and 3 results demonstrate that normal weight babies were born to mothers who consumed milk and curd daily, as well as pulses and beans. The daily intake of green leafy and vegetables were associated with a normal birth weight $21 \%$ in 2005-2006 and $19 \%$ in 2015-2016, respectively, compared to those who did not consume these items. In 2005-2006 and 2015-2016, the odds ratios for daily fruits intake were about $50 \%$ higher and for weekly fruits intake were $25 \%$ higher, indicating a strong positive effect on normal birth weight. Babies born to mothers who eat fish on a daily or weekly basis have a $60 \%$ and $11 \%$ greater chance of having a normal birth weight than babies born to mothers who never eat fish. Female babies had a higher chance of being born underweight, with percentages of $10 \%$ and $14 \%$ for NFHS 3 and 4 , respectively. In NFHS-3, the first-born infant was more likely to be born with normal weight than their higher birth order siblings, whereas in NFHS-4, the $2^{\text {nd }}-3^{\text {rd }}$ birth order $(22 \%)$ and $4^{\text {th }}$ birth order $(22 \%)$ birth orders had higher proportions of normal weight babies (17\%). In NFHS-3, 15\% of normal BMI mothers and 31\% of obese women had a better likelihood of giving birth to a normal birth weight infant than underweight mothers, but the percentages were $24 \%$ and $38 \%$ in NFHS-4, respectively. More than half of women with a secondary education in NFHS-3 and 19\% of mothers with a secondary education in NFHS-4 delivered babies with a normal birth weight than mothers without a secondary education. Children born to women in the middle and upper wealth category were more likely than their poorer counterparts to have a normal birth weight. In both the NFHS surveys, nonHindu and non-Muslim mothers of infants had a 30\% and 58\% share of normal birth weight babies, respectively. Children from larger households ( $\geq 9$ members) were $24 \%$ more likely to be born with LBW than the children from smaller families. In 2015-2016, institutional delivery had a significant effect on children's birth weight. About $15 \%$ of private and $22 \%$ of home deliveries were less likely to be born with the normal birth weight than public health institutional deliveries. Women with less than two dietary diversity categories had significantly higher proportion of low birth weight babies as compared to two or more dietary diversity categories (Table 3).

\section{Birth size}

Tables 2 and 3 also show that mothers who had consumed milk and curd on a daily or weekly basis were more likely to have babies of normal size at birth. Pregnant women who ate pulses and beans daily were $38 \%$ more likely in NFHS-3 and 10\% more likely in NFHS-4 to have babies of average or large size. In the 2005-2006 and 2015-2016 analyses, fish consumption demonstrated divergent effects on the size of the infant at birth. Fish eating by mothers on a daily basis was found to increase the proportion of average or above average births size by $21 \%$ compared to mothers who never or only occasionally consumed fish during NFHS-4. When compared to singletons, twins were more likely to be born with a shorter birth size. In NFHS-3, it was $19 \%$ higher, and in NFHS-4, it was $28 \%$ higher. The BMI of the mother has a significant effect on the size of the newborn infant. When compared to underweight mothers, babies born with the average or normal birth size were $29 \%$ more likely to be born to mothers who were overweight or obese. Between NHFS-3 and NHFS-4, the effect of the mother's BMI on birth size had decreased among normal BMI women but increased among obese women. The proportion of children born with average and above average birth sizes had increased with increasing wealth in both the rounds of the NFHS. In NFHS-4, for example, infants born to the richest wealth quintile were $59 \%$ more likely to have than those born to the lowest wealth quintile to born with the average or above birth size. Babies born at public health institutions had a higher proportion of average or above average birth weights than babies born in private health facilities or at home. Those babies who were born in private health facility and home, were $15 \%$ and $42 \%$, respectively, less likely to have average or above size at birth in comparison to birth in public health institution. There was less likely of small size of babies at birth with women's dietary diversity two or more compared to their counterparts.

\section{DISCUSSION}

This study illustrates the association between mothers' dietary pattern of intake and their children's birth weight and birth size, based on two recent NFHS surveys. With a distribution of birth weight, birth size, and sociodemographic characteristics, around four-fifths of women in NFHS-3 and more than four-fifths of women in NFHS-4 consumed these seven food groups daily, 
Table 2: Odds of normal birth weights and birth size varying by specific food intake among mothers in India, 2005-2016

\begin{tabular}{|c|c|c|c|c|c|c|c|c|c|}
\hline \multirow{3}{*}{$\begin{array}{l}\text { Background } \\
\text { characteristics }\end{array}$} & \multirow[t]{3}{*}{ Category } & \multicolumn{4}{|c|}{ Birth weight } & \multicolumn{4}{|c|}{ Birth size } \\
\hline & & \multicolumn{2}{|c|}{ NFHS-3 (2005-06) } & \multicolumn{2}{|c|}{ NFHS-4 (2015-16) } & \multicolumn{2}{|c|}{ NFHS-3 (2005-06) } & \multicolumn{2}{|c|}{ NFHS-4 (2015-16) } \\
\hline & & $\begin{array}{l}\text { Odds } \\
\text { ratio }\end{array}$ & $\begin{array}{c}\text { CI } \\
(95 \%)\end{array}$ & $\begin{array}{l}\text { Odds } \\
\text { ratio }\end{array}$ & $\begin{array}{c}\text { CI } \\
(95 \%)\end{array}$ & Odds ratio & $\underset{(95 \%)}{\text { CI }}$ & Odds ratio & $\begin{array}{c}\text { CI } \\
(95 \%)\end{array}$ \\
\hline
\end{tabular}

Milk and curd Never/

Foods items

\begin{tabular}{|c|c|c|c|c|c|c|c|c|c|}
\hline & \multicolumn{9}{|l|}{ occasionally $\mathbb{}$} \\
\hline & Daily & $1.03 *$ & $(0.97,1.10)$ & $0.93 *$ & $(0.86,1.00)$ & $1.03 * *$ & $(0.98,1.09)$ & $1.09 * *$ & $(1.02,1.17)$ \\
\hline & Weekly & $0.89 * * *$ & $(0.82,0.96)$ & $0.91 * *$ & $0.84,0.98)$ & 1.04 & $(0.98,1.12)$ & $1.04 *$ & $(0.96,1.12)$ \\
\hline \multirow[t]{3}{*}{$\begin{array}{l}\text { Pulses and } \\
\text { beans }\end{array}$} & $\begin{array}{l}\text { Never/ } \\
\text { occasionally® }\end{array}$ & & & & & & & & \\
\hline & Daily & $0.88 * *$ & $(0.81,0.97)$ & $1.02 *$ & $(0.92,1.14)$ & $1.38 * * *$ & $(1.28,1.48)$ & $1.10^{*}$ & $(1.00,1.21)$ \\
\hline & Weekly & $0.76^{* * *}$ & $(0.70,0.83)$ & $0.91 *$ & $(0.83,1.01)$ & $1.27 * * *$ & $(1.18,1.36)$ & $0.97 *$ & $(0.89,1.06)$ \\
\hline \multirow[t]{3}{*}{$\begin{array}{l}\text { Green leafy } \\
\text { vegetables }\end{array}$} & $\begin{array}{l}\text { Never/ } \\
\text { occasionally® }\end{array}$ & & & & & & & & \\
\hline & Daily & $1.21 * * *$ & $(1.09,1.35)$ & $1.19 * * *$ & $(1.08,1.30)$ & 0.95 & $(0.87,1.03)$ & $1.08 *$ & $(0.99,1.18)$ \\
\hline & Weekly & $0.97 *$ & $(0.87,1.08)$ & $1.07 * *$ & $(0.98,1.18)$ & 0.99 & $(0.91,1.08)$ & $1.03 * *$ & $(0.95,1.12)$ \\
\hline \multirow[t]{3}{*}{ Fruits } & $\begin{array}{l}\text { Never/ } \\
\text { occasionally }\end{array}$ & & & & & & & & \\
\hline & Daily & $1.50 * * *$ & $(1.38,1.63)$ & $1.01^{*}$ & $(0.90,1.13)$ & $1.04 * *$ & $(0.96,1.13)$ & $1.06^{*}$ & $(0.89,1.13)$ \\
\hline & Weekly & $1.25 * * *$ & $(1.18,1.33)$ & $1.09 * *$ & $(1.01,1.17)$ & $1.06^{* *}$ & $(0.95,1.06)$ & $0.96 * *$ & $(0.89,1.02)$ \\
\hline \multirow[t]{3}{*}{ Eggs } & $\begin{array}{l}\text { Never/ } \\
\text { occasionally }\end{array}$ & & & & & & & & \\
\hline & Daily & $1.31 * * *$ & $(1.15,1.50)$ & $1.01 *$ & $(0.84,1.22)$ & $1.01 *$ & $(0.89,1.15)$ & $1.09 *$ & $(0.90,1.33)$ \\
\hline & Weekly & $1.31 * * *$ & $(1.23,1.40)$ & $1.09 *$ & $(1.00,1.19)$ & $1.13^{* * *}$ & $(1.06,1.20)$ & $1.01^{*}$ & $(0.94,1.09)$ \\
\hline \multirow[t]{3}{*}{ Fish } & $\begin{array}{l}\text { Never/ } \\
\text { occasionally }\end{array}$ & & & & & & & & \\
\hline & Daily & $1.59 * * *$ & $(1.43,1.76)$ & $0.99 *$ & $(0.84,1.17)$ & 0.90 & $(0.82,1.01)$ & $1.21 *$ & $(1.01,1.46)$ \\
\hline & Weekly & $1.11^{* *}$ & $(1.03,1.20)$ & $0.97 *$ & $(0.88,1.06)$ & $0.84 * * *$ & $(0.78,0.89)$ & $0.97 *$ & $(0.89,1.06)$ \\
\hline \multirow[t]{4}{*}{ Meat } & $\begin{array}{l}\text { Never/ } \\
\text { occasionally® }\end{array}$ & & & & & & & & \\
\hline & Daily & $1.21 *$ & $(1.00,1.46)$ & $0.93^{*}$ & $(0.69,1.26)$ & $1.14^{*}$ & $(0.94,1.39)$ & $0.98^{*}$ & $(0.74,1.31)$ \\
\hline & Weekly & $1.16^{* * *}$ & $(1.08,1.24)$ & $1.04 * *$ & $(0.95,1.13)$ & $1.15^{* * *}$ & $(1.08,1.23)$ & $1.03 *$ & $(0.95,1.12)$ \\
\hline & \multicolumn{9}{|c|}{ Sociodemographic characteristics } \\
\hline \multirow[t]{2}{*}{ Sex of child } & Male ${ }^{\circledR}$ & & & & & & & & \\
\hline & Female & $0.90 * * *$ & $(0.85,0.94)$ & $0.86^{* * *}$ & $(0.81,0.92)$ & $090 * * *$ & $(0.86,0.94)$ & $0.94 * *$ & $(0.89,0.99)$ \\
\hline \multirow[t]{2}{*}{ Twin child } & $\mathrm{No} \circledast$ & & & & & & & & \\
\hline & Yes & $0.74 * * *$ & $(0.71,0.78)$ & $0.79 * * *$ & $(0.74,0.81)$ & $0.81 * * *$ & $(0.79,0.84)$ & $0.72 * * *$ & $(0.65,0.76)$ \\
\hline \multirow{3}{*}{$\begin{array}{l}\text { Birth order of } \\
\text { child }\end{array}$} & First ${ }^{\circledR}$ & & & & & & & & \\
\hline & $2^{\text {nd }}-3^{\text {rd }}$ & 0.99 & $(0.93,1.05)$ & $1.22 * * *$ & $(1.13,1.32)$ & $1.11 * * *$ & $(1.05,1.17)$ & $1.08 * *$ & $(1.01,1.15)$ \\
\hline & $4^{\text {th }}$ and above & $0.67 * * *$ & $(0.61,0.74)$ & $1.17 *$ & $(1.02,1.35)$ & 1.05 & $(0.97,1.14)$ & 0.99 & $(0.89,1.09)$ \\
\hline \multirow[t]{3}{*}{ BMI of women } & Underweight ${ }^{\circledR}$ & & & & & & & & \\
\hline & Normal & $1.15^{* * *}$ & $(1.08,1.22)$ & $1.24 * * *$ & $(1.16,1.33)$ & $1.15^{* * *}$ & $(1.09,1.21)$ & $1.07 * *$ & $(1.01,1.14)$ \\
\hline & $\begin{array}{l}\text { Overweight/ } \\
\text { obese }\end{array}$ & $1.31 * * *$ & $(1.19,1.43)$ & $1.38 * * *$ & $(1.24,1.53)$ & $1.24 * * *$ & $(1.12,1.36)$ & $1.29 * * *$ & $(1.17,1.44)$ \\
\hline \multirow[t]{3}{*}{ Age of mother } & $15-24$ years ${ }^{\circledR}$ & & & & & & & & \\
\hline & $25-34$ years & $1.11 * * *$ & $(1.04,1.17)$ & $1.08 *$ & $(1.01,1.16)$ & $1.20 * * *$ & $(1.14,1.27)$ & $1.08 * *$ & $(1.01,1.15)$ \\
\hline & $\begin{array}{l}35 \text { and above } \\
\text { years }\end{array}$ & 1.04 & $(0.92,1.16)$ & 1.07 & $(0.94,1.23)$ & $1.22 * * *$ & $(1.11,1.34)$ & $1.11 *$ & $(0.99,1.25)$ \\
\hline \multirow[t]{3}{*}{ Education } & Illiterate $\AA$ & & & & & & & & \\
\hline & Primary & $1.51 * * *$ & $(1.39,1.65)$ & 1.01 & $(0.92,1.12)$ & 0.99 & $(0.92,1.06)$ & 0.98 & $(0.90,1.07)$ \\
\hline & $\begin{array}{l}\text { Secondary and } \\
\text { above }\end{array}$ & $2.11 * * *$ & $(1.97,2.27$ & $1.19 * * *$ & $(1.09,1.30)$ & $1.15^{* * *}$ & $(1.08,1.22)$ & $1.07 *$ & $(1.00,1.16)$ \\
\hline
\end{tabular}


Table 2: (Continued)

\begin{tabular}{|c|c|c|c|c|c|c|c|c|c|}
\hline \multirow{3}{*}{$\begin{array}{l}\text { Background } \\
\text { characteristics }\end{array}$} & \multirow[t]{3}{*}{ Category } & \multicolumn{4}{|c|}{ Birth weight } & \multicolumn{4}{|c|}{ Birth size } \\
\hline & & \multicolumn{2}{|c|}{ NFHS-3 (2005-06) } & \multicolumn{2}{|c|}{ NFHS-4 (2015-16) } & \multicolumn{2}{|c|}{ NFHS-3 (2005-06) } & \multicolumn{2}{|c|}{ NFHS-4 (2015-16) } \\
\hline & & $\begin{array}{l}\text { Odds } \\
\text { ratio }\end{array}$ & $\begin{array}{c}\text { CI } \\
(95 \%)\end{array}$ & $\begin{array}{l}\text { Odds } \\
\text { ratio }\end{array}$ & $\begin{array}{c}\text { CI } \\
(95 \%)\end{array}$ & Odds ratio & $\begin{array}{c}\text { CI } \\
(95 \%)\end{array}$ & Odds ratio & $\begin{array}{c}\text { CI } \\
(95 \%)\end{array}$ \\
\hline \multirow{3}{*}{$\begin{array}{l}\text { Empowerment } \\
\text { level }\end{array}$} & Low ${ }^{\circledR}$ & & & & & & & & \\
\hline & Medium & 0.95 & $(0.89,1.02)$ & 1.04 & $(0.93,1.08)$ & $1.07 * *$ & $(1.01,1.13)$ & 1.01 & $(0.94,1.08)$ \\
\hline & High & 0.99 & $(0.93,1.06)$ & $1.11^{* *}$ & $(1.03,1.19)$ & $1.25 * * *$ & $(1.18,1.32)$ & $1.10 * *$ & $(1.02,1.18)$ \\
\hline \multirow[t]{5}{*}{ Wealth index } & Poorest $\AA$ & & & & & & & & \\
\hline & Poorer & $1.23 * * *$ & $(1.10,1.37)$ & 0.99 & $(0.90,1.09)$ & $1.07 *$ & $(1.01,1.15)$ & 1.05 & $(0.93,1.09)$ \\
\hline & Middle & $1.63 * * *$ & $(1.47,1.81)$ & 0.95 & $(0.85,1.04)$ & $1.12 * * *$ & $(1.04,1.21)$ & $1.13 * *$ & $(1.03,1.23)$ \\
\hline & Richer & $2.10 * * *$ & $(1.89,2.34)$ & 1.05 & $(0.89,1.13)$ & $1.24 * * *$ & $(1.14,1.35)$ & $1.16^{* *}$ & $(1.04,1.30)$ \\
\hline & Richest & $2.90 * * *$ & $(2.56,3.27)$ & $1.17 *$ & $(1.02,1.34)$ & $1.43 * * *$ & $(1.28,1.59)$ & $1.59 * * *$ & $(1.39,1.82)$ \\
\hline \multirow[t]{3}{*}{ Caste } & $\mathrm{SC} / \mathrm{ST} \circledast$ & & & & & & & & \\
\hline & $\mathrm{OBC}$ & 0.99 & $(0.92,1.06)$ & 1.05 & $(0.98,1.13)$ & $1.05^{*}$ & $(0.99,1.11)$ & $1.08 * *$ & $(1.01,1.15)$ \\
\hline & Other & $0.92 *$ & $(0.86,0.99)$ & $1.08 *$ & $(0.99,1.18)$ & 0.96 & $(0.90,1.02)$ & 1.02 & $(0.94,1.12)$ \\
\hline \multirow[t]{2}{*}{ Residence } & Rural $®$ & & & & & & & & \\
\hline & Urban & $1.06^{*}$ & $(1.00,1.13)$ & 0.92 & $(0.85,0.99)$ & 1.01 & $(0.95,1.07)$ & 0.98 & $(0.91,1.06)$ \\
\hline \multirow[t]{3}{*}{ Religion } & Hindu ${ }^{\circledR}$ & & & & & & & & \\
\hline & Muslims & $0.80 * * *$ & $(0.73,0.87)$ & 1.01 & $(0.91,1.12)$ & 1.06 & $(0.99,1.14)$ & 0.97 & $(0.89,1.06)$ \\
\hline & Other & $1.30 * * *$ & $(1.20,1.42)$ & $1.58 * * *$ & $(1.40,1.77)$ & 1.05 & $(0.98,1.13)$ & 0.98 & $(0.89,1.08)$ \\
\hline \multirow[t]{3}{*}{ Family size } & 0-4 members ${ }^{\circledR}$ & & & & & & & & \\
\hline & 5-8 members & 0.96 & $(0.90,1.03)$ & 1.05 & $(0.97,1.13)$ & $1.11 * * *$ & $(1.05,1.17)$ & 1.04 & $(0.97,1.11)$ \\
\hline & $\begin{array}{l}9 \text { and above } \\
\text { members }\end{array}$ & $0.76^{* * *}$ & $(0.70,0.82)$ & 1.04 & $(0.94,1.14)$ & $1.09 * *$ & $(1.02,1.17)$ & 0.97 & $(0.89,1.06)$ \\
\hline \multirow[t]{3}{*}{$\begin{array}{l}\text { Institutional } \\
\text { delivery }\end{array}$} & $\begin{array}{l}\text { Public } \\
\text { hospital }{ }^{\circ}\end{array}$ & & & & & & & & \\
\hline & $\begin{array}{l}\text { Private } \\
\text { hospital }\end{array}$ & $0.92 * *$ & $(0.86,0.98)$ & $0.89 * * *$ & $(0.83,0.96)$ & 1.01 & $(0.93,1.07)$ & $0.85^{* * *}$ & $(0.79,0.92)$ \\
\hline & Home & $0.96 * * *$ & $(0.93,0.99)$ & $0.78 * * *$ & $(0.70,0.87)$ & 1.01 & $(0.94,1.07)$ & $0.58 * * *$ & $(0.54,0.62)$ \\
\hline
\end{tabular}

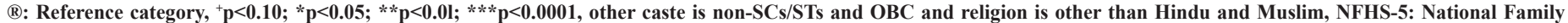
Health Survey-5, BMI: Body mass index, SC: Scheduled caste, ST: Scheduled tribe, OBC: Other backward class, CI: Confidence interval, LL: Lower limit, UL: Upper limit

weekly, or occasionally in their diet. According to the results of BLR model, mothers who consumed milk and curd daily basis were less likely in NFHS-4 as compared to NFHS-3, to have an infant who did not have a LBW or size in comparison to women who never consumed these food groups. The likelihood of weekly milk and curd intake increased from NFHS-3 to NFHS-4, and this association was found to be significant when birth weight was taken in account. The likelihood of normal birth weight babies is significant (around 15\% more from NFHS-3 to NFHS4) among those mothers who had daily intake of pulses and beans. Those mothers who had consume fruits and eggs in their daily diet were more likely to have normal delivery. Higher odds ratios of normal birth weight children were more likely among those mothers who were aged 25 years or above, secondary or more educated, or have medium or high empowerment level, or belong to richer or richest wealth quintile, and $\mathrm{OBCs} / \mathrm{Other}$ caste groups, other religious groups, and families with five or more members. Mothers who had consumed three or four foods daily in their daily diet intake had a higher likelihood of having a normal birth weight or size infant in both the NFHS-3 and NFHS-4 surveys. With more maternal education, a normal BMI, a higher wealth group, other religious group, and four or more birth order, the chances of having a normal birth weight and size increased. The previous researches have also shown that mother's diet intake has positive effect on the birth weight and size. This finding is consistent with prior research from India and other developing nations, and it reveals and validates that there is a strong association between child birth weight and size and the mother's intake of diverse food groups in the daily diet during pregnancy [1,14-18]. A study in Denmark found that infants born to mothers who ate processed meats, potatoes, and high dairy fats with a low intake of fruits and vegetables had the LBW, and similarly, study in South Australia also found that maternal dietary composition influences the fetal growth. In comparison to uneducated women, educated women have more access to health-care facilities and have more knowledgeable about the disadvantages of inadequate health-care utilization. Since, wealthy women are more likely to be educated, so they would visit more to better health-care facilities and be more informed about the risks of inadequate health care. Furthermore, wealthier families can afford healthy, nutritious, and variety of food groups throughout pregnancy. As a result, the risks of having a baby with a LBW and size are reduced among wealthier women. The outcomes of this study showed that women with a diet diversity score of more than 4 are at a 
Table 3: Odds of normal birth weights and birth size varying by diversity of food intake among mothers in India, 2005-2016

\begin{tabular}{|c|c|c|c|c|c|c|c|c|}
\hline \multirow{3}{*}{$\begin{array}{l}\text { Background } \\
\text { characteristics }\end{array}$} & \multicolumn{4}{|c|}{ Birth weight } & \multicolumn{4}{|c|}{ Birth size } \\
\hline & \multicolumn{2}{|c|}{ NFHS-3 (2005-06) } & \multicolumn{2}{|c|}{ NFHS-4 (2015-16) } & \multicolumn{2}{|c|}{ NFHS-3 (2005-06) } & \multicolumn{2}{|c|}{ NFHS-4 (2015-16) } \\
\hline & $\begin{array}{l}\text { Odds } \\
\text { ratio }\end{array}$ & $\begin{array}{c}(95 \% \text { CI }) \\
\text { (LL-UL) }\end{array}$ & $\begin{array}{l}\text { Odds } \\
\text { ratio }\end{array}$ & $\begin{array}{c}\text { (95\% CI) } \\
\text { (LL-UL) }\end{array}$ & $\begin{array}{l}\text { Odds } \\
\text { ratio }\end{array}$ & $\begin{array}{l}\text { (95\% CI) } \\
\text { (LL-UL) }\end{array}$ & $\begin{array}{l}\text { Odds } \\
\text { ratio }\end{array}$ & $\begin{array}{c}\text { (95\% CI) } \\
\text { (LL-UL) }\end{array}$ \\
\hline \multicolumn{9}{|l|}{ Diet pattern } \\
\hline \multicolumn{9}{|l|}{ No any® } \\
\hline One & 1.03 & $(0.95,1.12)$ & $1.27 * * *$ & $(1.11,1.45)$ & 0.98 & $(0.92,1.05)$ & 1.07 & $(0.98,1.17)$ \\
\hline Two & $1.14^{* *}$ & $(1.04,1.24)$ & $1.32 * * *$ & $(1.16,1.51)$ & $1.06^{*}$ & $(0.99,1.13)$ & $1.21 * * *$ & $(1.11,1.33)$ \\
\hline Three & $1.27 * * *$ & $(1.15,1.41)$ & $1.44 * * *$ & $(1.25,1.66)$ & $1.09 * *$ & $(1.01,1.18)$ & $1.26^{* * *}$ & $(1.12,1.41)$ \\
\hline Four and above & $1.15^{* *}$ & $(1.00,1.33)$ & $1.59 * * *$ & $(1.36,1.87)$ & $1.19 * * *$ & $(1.06,1.33)$ & $1.41 * * *$ & $(1.18,1.69)$ \\
\hline \multicolumn{9}{|l|}{ Sex of child } \\
\hline \multicolumn{9}{|l|}{ Male $\AA$} \\
\hline Female & $0.87 * * *$ & $(0.82,0.92)$ & 0.87 & $(0.81,0.93)$ & $0.90 * * *$ & $(0.86,0.94)$ & $0.87 * * *$ & $(0.82,0.93)$ \\
\hline \multicolumn{9}{|l|}{ Twin child } \\
\hline \multicolumn{9}{|l|}{$\mathrm{No} \circledast$} \\
\hline Yes & $0.87 * * *$ & $(0.85,0.89)$ & $0.79 * * *$ & $(0.75,0.84)$ & $0.81 * * *$ & $(0.77,0.83)$ & $0.78 * * *$ & $(0.73,0.83)$ \\
\hline \multicolumn{9}{|l|}{ Birth order } \\
\hline \multicolumn{9}{|l|}{$1^{\mathrm{st} \circledast}$} \\
\hline $2^{\text {nd }}-3^{\text {rd }}$ & $1.21 * * *$ & $(1.13,1.29)$ & 1.11 & $(1.03,1.21)$ & $1.11 * * *$ & $(1.05,1.17)$ & $1.09 * *$ & $(1.00,1.18)$ \\
\hline $4^{\text {th }}$ and above & $1.12 *$ & $(0.99,1.25)$ & 1.10 & $(0.95,1.28)$ & 1.06 & $(0.98,1.15)$ & 1.01 & $(0.89,1.13)$ \\
\hline \multicolumn{9}{|l|}{ BMI of women } \\
\hline \multicolumn{9}{|l|}{ Underweight ${ }^{\circledR}$} \\
\hline Normal & $1.24 * * *$ & $(1.15,1.33)$ & 1.32 & $(1.21,1.43)$ & $1.14 * *$ & $(1.09,1.20)$ & $1.11 * *$ & $(1.02,1.19)$ \\
\hline $\begin{array}{l}\text { Overweight/ } \\
\text { obese }\end{array}$ & $1.38 * * *$ & $(1.24,1.53)$ & 1.44 & $(1.28,1.63)$ & $1.23 * * *$ & $(1.12,1.35)$ & $1.33 * * *$ & $(1.18,1.50)$ \\
\hline \multicolumn{9}{|l|}{ Age } \\
\hline \multicolumn{9}{|l|}{ 15-24 years ${ }^{\circledR}$} \\
\hline 25-34 years & $1.08 * *$ & $(1.01,1.16)$ & 1.21 & $(1.12,1.32)$ & $1.19 * * *$ & $(1.13,1.26)$ & $1.06^{* *}$ & $(0.98,1.15)$ \\
\hline $\begin{array}{l}35 \text { and above } \\
\text { years }\end{array}$ & 1.07 & $(0.94,1.22)$ & 1.27 & $(1.08,1.50)$ & $1.18^{* * *}$ & $(1.08,1.30)$ & $1.10^{* *}$ & $(0.97,1.26)$ \\
\hline \multicolumn{9}{|l|}{ Education } \\
\hline \multicolumn{9}{|l|}{ Illiterate ${ }^{\circledR}$} \\
\hline Primary & 1.02 & $(0.92,1.13)$ & 1.06 & $(0.94,1.21)$ & 0.98 & $(0.92,1.05)$ & 0.99 & $(0.89,1.10)$ \\
\hline $\begin{array}{l}\text { Secondary and } \\
\text { above }\end{array}$ & $1.21 * * *$ & $(1.12,1.32)$ & $1.38 * * *$ & $(1.24,1.54)$ & $1.14 * *$ & $(1.07,1.21)$ & $1.07 *$ & $(0.98,1.17)$ \\
\hline \multicolumn{9}{|l|}{$\begin{array}{l}\text { Empowerment } \\
\text { level }\end{array}$} \\
\hline Low ${ }^{\circledR}$ & & & & & & & & \\
\hline Medium & 1.01 & $(0.93,1.08)$ & $0.92 *$ & $(0.84,1.01)$ & $1.07 * *$ & $(1.02,1.13)$ & 0.98 & $(0.90,1.06)$ \\
\hline High & $1.11 * *$ & $(1.03,1.19)$ & 0.98 & $(0.89,1.07)$ & $1.26^{* * *}$ & $(1.19,1.33)$ & $1.10 * *$ & $(1.01,1.19)$ \\
\hline Wealth category & & & & & & & & \\
\hline Poorest $\AA$ & & & & & & & & \\
\hline Poorer & 0.98 & $(0.90,1.08)$ & 0.99 & $(0.82,1.19)$ & $1.09 * *$ & $(1.02,1.17)$ & 1.02 & $(0.93,1.13)$ \\
\hline Middle & 0.93 & $(0.85,1.03)$ & 0.96 & $(0.80,1.14)$ & $1.15^{* * *}$ & $(1.07,1.24)$ & $1.13 * *$ & $(1.02,1.26)$ \\
\hline Richer & $0.98^{*}$ & $(0.88,1.10)$ & 0.99 & $(0.83,1.18)$ & $1.30 * * *$ & $(1.19,1.41)$ & $1.14 * * *$ & $(1.01,1.29)$ \\
\hline Richest & $1.12^{*}$ & $(0.98,1.28)$ & $1.13^{* *}$ & $(0.93,1.37)$ & $1.50 * * *$ & $(1.35,1.66)$ & $1.63 * * *$ & $(1.40,1.91)$ \\
\hline Caste & & & & & & & & \\
\hline $\mathrm{SC} / \mathrm{ST} \AA$ & & & & & & & & \\
\hline $\mathrm{OBC}$ & 1.02 & $(0.95,1.10)$ & 1.02 & $(0.92,1.13)$ & $1.05 *$ & $(0.99,1.11)$ & $1.06^{*}$ & $(0.98,1.14)$ \\
\hline Other & 1.06 & $(0.96,1.16)$ & $0.88 * *$ & $(0.80,0.98)$ & $0.95 *$ & $(0.89,1.01)$ & 1.03 & $(0.91,1.11)$ \\
\hline
\end{tabular}


Table 3: (Continued)

\begin{tabular}{|c|c|c|c|c|c|c|c|c|}
\hline \multirow{3}{*}{$\begin{array}{l}\text { Background } \\
\text { characteristics }\end{array}$} & \multicolumn{4}{|c|}{ Birth weight } & \multicolumn{4}{|c|}{ Birth size } \\
\hline & \multicolumn{2}{|c|}{ NFHS-3 (2005-06) } & \multicolumn{2}{|c|}{ NFHS-4 (2015-16) } & \multicolumn{2}{|c|}{ NFHS-3 (2005-06) } & \multicolumn{2}{|c|}{ NFHS-4 (2015-16) } \\
\hline & $\begin{array}{l}\text { Odds } \\
\text { ratio }\end{array}$ & $\begin{array}{l}(95 \% \text { CI }) \\
(\text { LL-UL) }\end{array}$ & $\begin{array}{l}\text { Odds } \\
\text { ratio }\end{array}$ & $\begin{array}{l}(95 \% \text { CI }) \\
(\text { LL-UL) }\end{array}$ & $\begin{array}{l}\text { Odds } \\
\text { ratio }\end{array}$ & $\begin{array}{l}(95 \% \text { CI }) \\
\text { (LL-UL) }\end{array}$ & $\begin{array}{l}\text { Odds } \\
\text { ratio }\end{array}$ & $\begin{array}{l}(95 \% \text { CI }) \\
(\text { LL-UL) }\end{array}$ \\
\hline \multicolumn{9}{|l|}{ Residence } \\
\hline \multicolumn{9}{|l|}{ Rural® } \\
\hline Urban & $0.93 *$ & $(0.86,1.00)$ & 0.99 & $(0.91,1.07)$ & 1.02 & $(0.96,1.08)$ & 0.96 & $(0.88,1.05)$ \\
\hline \multicolumn{9}{|l|}{ Religion } \\
\hline \multicolumn{9}{|l|}{ Hindu $\mathbb{}$} \\
\hline Muslims & 1.03 & $(0.94,1.14)$ & $1.17 * * *$ & $(1.05,1.32)$ & $1.08 * *$ & $(1.01,1.16)$ & $0.94 *$ & $(0.85,1.03)$ \\
\hline Other & $1.63 * * *$ & $(1.45,1.83)$ & $1.47 * * *$ & $(1.30,1.66)$ & 0.99 & $(0.93,1.07)$ & 1.09 & $(0.98,1.22)$ \\
\hline \multicolumn{9}{|l|}{ Family size } \\
\hline \multicolumn{9}{|l|}{ 0-4 members ${ }^{\circledR}$} \\
\hline 5-8 members & 1.03 & $(0.96,1.11)$ & 1.04 & $(0.95,1.13)$ & $1.11 * * *$ & $(1.05,1.17)$ & 1.02 & $(0.94,1.10)$ \\
\hline $\begin{array}{l}9 \text { and above } \\
\text { members }\end{array}$ & 1.01 & $(0.92,1.11)$ & 0.90 & $(0.81,1.01)$ & $1.10^{* * *}$ & $(1.03,1.18)$ & 0.94 & $(0.85,1.05)$ \\
\hline \multicolumn{9}{|l|}{$\begin{array}{l}\text { Institutional } \\
\text { delivery }\end{array}$} \\
\hline \multicolumn{9}{|l|}{$\begin{array}{l}\text { Public } \\
\text { hospital } \AA\end{array}$} \\
\hline Private hospital & $0.88 * * *$ & $(0.82,0.95)$ & 0.94 & $(0.87,1.02)$ & $1.01 *$ & $(0.94,1.08)$ & $0.86^{* * *}$ & $(0.78,0.94)$ \\
\hline Home & $0.79 * * *$ & $(0.71,0.88)$ & 0.97 & $(0.86,1.09)$ & $1.01 *$ & $(0.95,1.07)$ & $0.57 * * *$ & $(0.53,0.62)$ \\
\hline
\end{tabular}

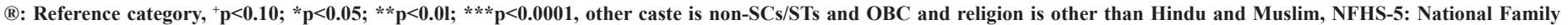
Health Survey-5, BMI: Body mass index, SC: Scheduled caste, ST: Scheduled tribe, OBC: Other backward class, CI: Confidence interval, LL: Lower limit, UL: Upper limit

lower risk of having LBW and smaller size of baby [8,19-21]. Dietary intake during pregnancy was observed to be significantly associated with the babies' birth weight or size. Poor nutrition and inadequate supplementation during pregnancy may cause placental growth to be slowed, as well as a reduction in the birth weight and size of the infants.

The results of this study should be interpreted with caution due to various limitations. The information on a child's birth weight or size was collected from a written record or from the mother's recall. As a result, the information from the mother's recall is prone to recall bias. Nonetheless, to minimize the recall bias in the study, we limited our sample to women who had given birth in the 5 years before to the survey. Furthermore, many occurrences of missing birth weight data exist because many children were not weighed at birth or respondents failed to respond. Due to the cross-sectional nature of the study design, we would be unable to assess the causal relationship between the outcome variable and the predictors. Longitudinal data would be needed in the future to further understand the mechanism behind India's high prevalence of LBW or small size children. Despite these limitations, this study gives valuable insights into maternal characteristics related with child LBW or small birth size, which may be relevant for policymaking in the Indian context to minimize the incidence of LBW and small birth size.

\section{CONCLUSION}

This study provides compelling evidence that mothers' diet patterns reflect nutritional status of newborns in various contexts in India. Interventions are needed for adequate utilization of $\mathrm{MCH}$ services and uptake of balanced energy-protein diet among pregnant mothers. Besides, special attention should be given to the socioeconomically disadvantaged women to address adverse pregnancy and birth outcomes. As a result, educational interventions must be offered in addition to the supplemental diverse nutrition support programs to achieve the sustainable development goals for India sooner.

\section{REFERENCES}

1. Swaminathan S, Hemalatha R, Pandey A, Kassebaum NJ, Laxmaiah A, Longvah $\mathrm{T}$, et al. The burden of child and maternal malnutrition and trends in its indicators in the states of India: The global burden of disease study 1990-2017. Lancet Child Adolesc Health 2019;3:855-70.

2. Kumar P, Singhal N. Mapping neonatal and under-5 mortality in India. Lancet 2020;395:1591-93.

3. Million Death Study Collaborators. Causes of neonatal and child mortality in India: A nationally representative mortality survey. Lancet 2010;376:1853-60.

4. International Institute for Population Sciences. National Family Health Survey (NFHS-4), 2015-16. Mumbai, India: International Institute for Population Sciences; 2017. p. 791-846.

5. Sethi V, de Wagt A, Bhanot A, Singh KD, Agarwal P, Murira Z, et al. Levels and determinants of malnutrition among India's urban poor women: An analysis of demographic health surveys 2006 and 2016. Mater Child Nutr 2020;16:e12978.

6. Lu MS, Chen QZ, He JR, Wei XL, Lu JH, Li SH, et al. Maternal dietary patterns and fetal growth: A large prospective cohort study in China. Nutrients 2016;8:257.

7. Durrani AM, Rani A. Effect of maternal dietary intake on the weight of the newborn in Aligarh city, India. Niger Med J 2011;52:177-81.

8. Zerfu TA, Umeta M, Baye K. Dietary diversity during pregnancy is associated with reduced risk of maternal anemia, preterm delivery, and low birth weight in a prospective cohort study in rural Ethiopia. Am J Clin Nutr 
2016;103:1482-8.

9. Zaveri A, Paul P, Saha J, Barman B, Chouhan P. Maternal determinants of low birth weight among Indian children: Evidence from the National Family Health Survey-4, 2015-16. PLoS One 2020;15:e0244562.

10. Rahman MS, Howlader T, Masud MS, Rahman ML. Association of lowbirth weight with malnutrition in children under five years in Bangladesh: Do mother's education, socio-economic status, and birth interval matter? PLoS One 2016;11:e0157814.

11. Acharya O, Zotor FB, Chaudhary P, Deepak K, Amuna P, Ellahi B. Maternal nutritional status, food intake and pregnancy weight gain in Nepal. J Health Manag 2016;18:1-12.

12. Verma S, Shrivastava R. Effect of maternal nutritional status on birth weight of baby. J Contemp Med Res 2016;3:943-5.

13. Marangoni F, Cetin I, Verduci E, Canzone G, Giovannini M, Scollo P, et al. Maternal diet and nutrient requirements in pregnancy and breastfeeding. An Italian consensus document. Nutrients 2016;8:629.

14. Akbari Z, Mansourian M, Kelishadi R. Relationship of the intake of different food groups by pregnant mothers with the birth weight and gestational age: Need for public and individual educational programs. J Educ Health Promot 2015;4:1-6.

15. Hwang J, Shin D, Kim H, Kwon O. Association of maternal dietary patterns during pregnancy with small-for-gestational-age infants: Korean mothers and children's environmental health (MOCEH) study. Am J Clin Nutr 2021;114:nqab340.

16. Rao S, Yajnik CS, Kanade A, Fall CH, Margetts BM, Jackson A, et al. Intake of micronutrient-rich foods in rural Indian mothers is associated with the size of their babies at birth: Pune maternal nutrition study. J Nutr 2001;131:1217-24.

17. Starling AP, Sauder KA, Kaar JL, Shapiro AL, Siega-Riz AM, Dabelea D. Maternal dietary patterns during pregnancy are associated with newborn body composition. J Nutr 2017;147:1334-9.

18. Moore VM, Davies MJ, Willson KJ, Worsley A, Robinson JS. Dietary composition of pregnant women is related to size of the baby at birth. J Nutr 2004; $134: 1820-6$.

19. Bhatia J, Bhutta ZA, Kalhan SC, editors. Maternal and Child Nutrition: The First 1,000 Days. Vol. 1. Basel, Switzerland: Karger Medical and Scientific Publishers; 2013. p. 1-233.

20. Lassi ZS, Majeed A, Rashid S, Yakoob MY, Bhutta ZA. The interconnections between maternal and newborn health evidence and implications for policy. J Matern Fetal Neonatal Med 2013;26:3-53.

21. Jamshed S, Farah-Khan AB, Ali BB, Akram Z, Ariff M. Frequency of low birth weight and its relationship with maternal nutritional and dietary factors: A cross-sectional study. Cureus 2020;12:e8731.

Funding: None; Conflicts of Interest: None Stated.

How to cite this article: Brajesh, Shekhar C. Association of maternal dietary patterns with child birth weight and size in India: Evidence from National Family Health Survey, 2005 to 2016. Indian J Child Health. 2021; 8(12):416-425. 